\title{
ADHD, Smoking Withdrawal, and Inhibitory Control: Results of a Neuroimaging Study with Methylphenidate Challenge
}

\author{
Maggie M Sweitzer,, , Scott H Kollins', Rachel V Kozink', Matt Hallyburton', Joseph English', \\ Merideth A Addicott', Jason A Oliver' and F Joseph McClernon' \\ 'Department of Psychiatry and Behavioral Sciences, Duke University School of Medicine, Durham, NC, USA
}

\begin{abstract}
Smoking withdrawal negatively impacts inhibitory control, and these effects are greater for smokers with preexisting attention problems, such as attention deficit/hyperactivity disorder (ADHD). The current study preliminarily evaluated changes in inhibitory control-related behavior and brain activation during smoking withdrawal among smokers with ADHD. Moreover, we investigated the role of catecholamine transmission in these changes by examining the effects of $40 \mathrm{mg}$ methylphenidate (MPH) administration. Adult daily smokers with $(n=17)$ and without $(n=20)$ ADHD completed $\mathrm{MRR}$ scanning under each of three conditions: (a) smoking as usual +placebo; (b) $24 \mathrm{~h}$ smoking abstinence+placebo and (c) $24 \mathrm{~h}$ smoking abstinence+MPH. Scan order was randomized and counterbalanced. Participants completed a modified Go/No-Go task to assess both sustained and transient inhibitory control. Voxelwise analysis of task-related BOLD signal revealed a significant group-by-abstinence interaction in occipital/parietal cortex during sustained inhibition, with greater abstinence-induced decreases in activation observed among ADHD smokers compared with non-ADHD smokers. Changes in behavioral performance during abstinence were associated with changes in activation in regions of occipital and parietal cortex and bilateral insula during sustained inhibition in both groups. MPH administration improved behavioral performance and increased sustained inhibitory control-related activation for both groups. During transient inhibition, MPH increased prefrontal activation for both groups and increased striatal activation only among ADHD smokers. These preliminary findings suggest that abstinence-induced changes in catecholamine transmission in visual attention areas (eg, occipital and superior parietal cortex) may be associated with inhibitory control deficits and contribute to smoking vulnerability among individuals with ADHD.

Neuropsychopharmacology (20 I8) 43, 85 I-858; doi:I0.1038/npp.20 I7.248; published online I5 November 2017
\end{abstract}

\section{INTRODUCTION}

Attention deficit/hyperactivity disorder (ADHD) is a commonly diagnosed disorder affecting approximately $5-7 \%$ of children and $4 \%$ of adults in the United States (Kessler et al, 2006; Willcutt, 2012). Individuals with ADHD smoke at higher rates than the general population (Molina and Pelham, 2003; Kessler et al, 2006), initiate smoking at an earlier age (Milberger et al, 1997), and have higher levels of nicotine dependence (Pomerleau et al, 1995). Furthermore, adults with ADHD report greater craving and withdrawal symptoms upon quitting smoking (McClernon et al, 2011) and are more likely to relapse (Humfleet et al, 2005).

One hypothesis for the strong links between ADHD and smoking is that deficits of inhibitory control-or the ability to withhold prepotent/overlearned responses-may contribute to increased smoking reinforcement and relapse in this patient population (McClernon and Kollins, 2008a). Inhibitory control deficits are a central defining feature of ADHD

*Correspondence: Dr MM Sweitzer, Department of Psychiatry and Behavioral Sciences, Duke University Medical Center, 2608 Erwin Road., Suite 300, Durham, NC 277I0, USA, Tel: + I 919668 0094, Fax: + I 91968 1 0016, E-mail: maggie.sweitzer@duke.edu

Received 5 June 2017; revised 30 August 2017; accepted 9 October 2017; accepted article preview online 20 October 2017
(Barkley, 1997). Moreover, smoking abstinence worsens inhibitory control (Kozink et al, 2010; Ashare and Hawk, 2012; Kollins et al, 2013), and abstinence-induced deficits in inhibitory control have been shown to predict relapse (Powell et al, 2010; Froeliger et al, 2017). Importantly, inhibitory control deficits associated with smoking abstinence may be exacerbated among smokers with ADHD. In one study, overnight abstinence resulted in worse inhibitory control among ADHD as compared with non-ADHD smokers (McClernon et al, 2008b). Furthermore, smokers with ADHD exhibited greater smoking-reinforced responding (measured via Progressive Ratio Task) during abstinence compared with non-ADHD smokers, and smokingreinforced responding was correlated with abstinenceinduced deficits in inhibitory control (Kollins et al, 2013). As such, individuals with ADHD may be more reliant on smoking than non-ADHD smokers to avoid abstinenceinduced deficits in inhibitory control, and further, abstinence-induced deficits may make avoidance of relapse more difficult.

Despite documenting the effects of smoking abstinence on inhibitory control deficits among ADHD smokers, little is known about the neurobiology underlying these effects. fMRI and PET studies suggest that inhibitory control is subserved by dopaminergic circuits in the striatum and 
cortical regions, including the anterior cingulate cortex (ACC) and right inferior frontal gyrus (rIFG) (Chikazoe et al, 2009; Congdon et al, 2010; Ghahremani et al, 2012). Individuals with $\mathrm{ADHD}$ are thought to exhibit altered dopamine transmission as evidenced by reduced dopamine D2/D3 receptor availability and attenuated dopamine receptor binding following MPH administration within the striatum and midbrain (Volkow et al, 2007, 2009). In addition, multiple fMRI studies have demonstrated attenuated frontostriatal activation during inhibitory control (Cubillo et al, 2012; Hart et al, 2013) or increased activation in parietal or cerebellar regions, suggesting recruitment of compensatory attentional processes among children and adults with ADHD relative to healthy controls (Dillo et al, 2010). Nicotine withdrawal is also associated with perturbation of dopaminergic neurotransmission (Zhang et al, 2012) and changes in task-related brain activation in executive control regions, including the rIFG, dorsolateral prefrontal cortex (DLPFC), and ACC (Ashare et al, 2014; McClernon et $a l, 2015)$. In a previous study of non-ADHD smokers, we observed that smoking abstinence decreased inhibitory control and event-related activation in rIFG (Kozink et al, 2010). Based on these results, we hypothesized that smoking abstinence further decreases inhibitory control among $\mathrm{ADHD}$ smokers through its influence on relevant circuitry including the rIFG and ACC.

The present study was designed to evaluate the influence of smoking abstinence on behavioral and neural correlates of inhibitory control among smokers with and without ADHD. Participants were carefully screened to identify daily smokers who met diagnostic criteria for ADHD (ADHD Group) or who did not exhibit any DSM-IV Axis I disorder aside from nicotine dependence (Control Group). Three fMRI scans were conducted: once following smoking as usual (satiated) and twice following $24 \mathrm{~h}$ smoking abstinence. In one of the abstinence conditions, participants were scanned following administration of $40 \mathrm{mg}$ methylphenidate $(\mathrm{MPH})$; the other followed administration of placebo. During scanning, participants completed a modified Go/No-Go task that allows for assessment of both sustained and transient neural correlates of inhibitory control. We hypothesized that smoking abstinence would differentially alter inhibitory control behavior and neural activation in smokers with $\mathrm{ADHD}$ and that MPH would counteract these changes.

\section{MATERIALS AND METHODS}

\section{Overview of Study Design}

Participants completed five total sessions. First, a screening session was conducted, during which participants read and signed an Institutional Review Board approved consent form, and eligibility was determined. Participants then completed an fMRI training and medical safety session, which assessed tolerability of MPH effects and provided task training and acclimation to the scanner environment. Participants then completed three separate fMRI sessions under the following conditions: (1) Smoking as usual +placebo pill (SAT+PLAC); (2) $24 \mathrm{~h}$ abstinent+placebo pill (ABS+PLAC); and (3) $24 \mathrm{~h}$ abstinent+40 mg methylphenidate $(\mathrm{ABS}+\mathrm{MPH})$. FMRI sessions were held a minimum of 4 days apart $(M=10.7, \quad \mathrm{SD}=6.4)$, and order was counterbalanced and randomly assigned. Participants were compensated up to $\$ 940$ for completing all sessions.

\section{Participants}

Participants were right-handed, non-treatment-seeking smokers recruited from the community who were in good general health (determined by medical evaluation), smoked at least 10 cigarettes per day, had expired carbon monoxide $(\mathrm{CO}) \geqslant 10$ or positive urine cotinine $\geqslant 100 \mu \mathrm{l} / \mathrm{ml}$, and met additional DSM-IV diagnostic criteria for ADHD (ADHD Group) or no diagnoses aside from nicotine dependence (Control Group) as determined by a comprehensive psychiatric evaluation conducted by a $\mathrm{PhD}$-level psychologist (see Supplementary Methods and Materials). Participants were excluded if they had a current primary psychiatric disorder other than ADHD or nicotine dependence or current use of psychoactive medications (other than ADHD pharmacotherapies among ADHD participants). Participants currently receiving pharmacotherapy for ADHD $(n=5$ Adderall, $n=1$ Concerta+MPH prn) were eligible to participate if they agreed, in consultation with their medical provider, to a washout period of at least five times the halflife of their current medication prior to the training session and for the duration of participation. See Supplementary Methods and Materials for additional exclusion criteria.

\section{Study Drug and Medication Safety}

All participants completed a 4-h MPH safety session prior to scanning, during which $\mathrm{MPH}$ was administered via $40 \mathrm{mg}$ capsule, and vitals and self-reported symptoms were assessed to ensure safety and tolerability (see Supplementary Methods and Materials for additional details). During the imaging sessions, drug was administered under partial double-blind conditions. Participants were provided with a blinded capsule (MPH or placebo) at all imaging sessions. Experimenters were aware that the capsule was a placebo in the satiated condition but were unaware of the capsule condition in the abstinent conditions.

\section{fMRI Scanning Sessions}

At the start of each scanning session, breath $\mathrm{CO}$ was obtained ( $\mathrm{CO} \leqslant 5$ was required for abstinent sessions) and $\mathrm{MPH}$ or placebo drug was administered. After $75 \mathrm{~min}$, participants were escorted to the fMRI suite. In the satiated condition, participants also smoked one usual brand cigarette approximately $30 \mathrm{~min}$ prior to scanning. Immediately prior to entering the scanner, participants completed a modified version of the Shiffman-Jarvik Withdrawal Questionnaire (Shiffman and Jarvik, 1976).

\section{Inhibitory Control Task}

We used a modified version of a Go/No-Go task (see Figure 1) developed and validated by Chikazoe et al (2009). Unlike standard Go/No-Go tasks, this task features both frequent and rare Go stimuli, which allows for an evaluation of brain activation in response to No-Go stimuli (which are rare), controlling for Rare-Go trials, thus isolating inhibitory control from novelty detection. Stimuli consisted of two 


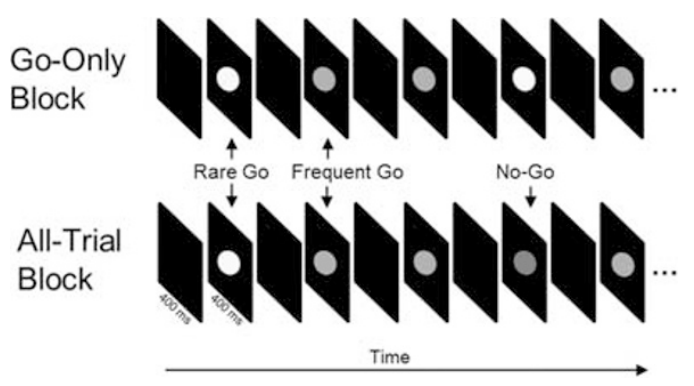

Figure I Example of stimuli presented during Go-Only and All-Trial Blocks of the Go/No-Go task. Stimuli are presented for $400 \mathrm{~ms}$, with $400 \mathrm{~ms}$ intertrial interval. Stimuli were presented in blocks, each lasting $44.8 \mathrm{~s}$ and consisting of 56 trials. Go-Only blocks include frequent Go stimuli (gray circles, $75 \%$ of trials) and rare Go stimuli (yellow circles, $25 \%$ of trials). All-Trial Blocks include frequent Go stimuli (gray circles, $75 \%$ of trials), rare Go stimuli (yellow circles, 12.5\% of trials), and No-Go stimuli (blue circles, 12.5\% of trials). Colors of rare Go and No-Go stimuli were counterbalanced across participants. A full color version of this figure is available at the Neuropsychopharmacology journal online.

different Go signals (frequent $=$ gray circles and rare $=$ yellow or blue circles) and a No-Go signal (blue or yellow circles). Participants were randomly assigned to either 'blue' or 'yellow' versions of the task, denoting which color stimulus represented the Rare-Go vs No-Go signals; once assigned, colors were held constant across sessions for each participant. In our modified version, stimuli were presented in two different block types in order to also evaluate sustained inhibitory control: In Go-Only (GO) Blocks, stimuli were Frequent-Go ( $75 \%$ of trials) and Rare-Go (25\% of trials) trials. In All-Trial (AT) Blocks, stimuli were Frequent-Go (75\%), Rare-Go (12.5\%), and No-Go (12.5\%) trials. Each block consisted of 56 trials and was completed 3 times per run. Blocks were interleaved with 13-s fixation and 2-s instructions indicating 'All Trials' or 'Go Only'. Participants completed two 6.25-min runs.

\section{fMRI Acquisition}

BOLD functional MR images were acquired using a $3 \mathrm{~T}$ GE Signal EXCITE HD scanner equipped with $40 \mathrm{mT} / \mathrm{m}$ gradients (Waukesha, WI). A gradient-recalled inward spiral pulse imaging sequence (SENSE spiral) collected 34 interleaved slices (TR $=1.5 \mathrm{~s}, \mathrm{TE}=30 \mathrm{~ms}$, flip angle $=60^{\circ}$, acquisition matrix $=64 \times 64$, field of view $=24.0 \mathrm{~cm}^{2}$, voxel size $\left.=3.75 \times 3.75 \times 4 \mathrm{~mm}^{3}\right)$. Participants completed a 5 -min resting state scan (reported separately) prior to Go/No-Go functional runs. After functional data collection, a T1weighted 3D structural image was acquired with a fast spoiled-gradient recalled sequence for 162 slices $(\mathrm{TR}=7.584 \mathrm{~ms}, \mathrm{TE}=2.936$, flip angle $=12$, acquisition matrix $=256 \times 256$, and voxel size $=1 \times 1 \times 1$ ).

\section{fMRI Data Analysis}

Images underwent preprocessing in FSL version 5.0.1: (1) rigid-body motion correction using MCFLIRT, (2) slice timing correction, (3) high-pass filtering, (4) registration to standard space using FLIRT, and (5) spatial smoothing using an $8 \mathrm{~mm}$ FWHM Gaussian kernel.
Sustained inhibitory control. Each block type (AT, GO) was modeled as a boxcar function convolved with double- $\gamma$ hemodynamic response function. A contrast image was created for $\mathrm{AT}>\mathrm{GO}$ blocks in order to isolate brain activation specific to sustained inhibitory control. Secondlevel models created a mean contrast image across both runs for each participant.

Transient inhibitory control. Events were modeled at the time of event onset convolved with a double $\gamma$ hemodynamic response function. Standard motion parameters and two nuisance regressors were included in the model: errors of commission on No-Go trials and correctly identified RareGo stimuli during the GO blocks. Two regressors of interest in the AT blocks (correctly identified No-Go and Rare-Go stimuli) were contrasted and carried forward to hypothesis testing. In order to be classified as correct, No-Go stimuli were required to be preceded by a correct Go trial. FrequentGo stimuli across both block types served as the baseline.

Hypothesis testing. In order to increase the interpretability of complex interaction effects in the absence of a fullfactorial design, both event-related and block contrasts were entered into two separate 2 (Group) $\times 2$ (Condition) ANOVAs using mixed effects (Flame1) analysis in FSL. First, effects of abstinence were examined by comparing SAT + PLAC with ABS+PLAC conditions. In a second model, effects of MPH during abstinence were examined by comparing $\mathrm{ABS}+\mathrm{MPH}$ with $\mathrm{ABS}+\mathrm{PLAC}$ conditions. Thresholding for significance was defined as $Z=2.3$, with cluster correction of $P<0.05$ across the whole brain.

\section{RESULTS}

\section{Participant Characteristics}

A total of 67 participants passed all inclusion and exclusion criteria and were enrolled in the study. Of these, 35 subjects ( $n=17$ ADHD; $n=18$ controls) completed all sessions and provided usable data on primary outcomes (see Supplementary Methods and Materials for details on exclusions). ADHD and Control groups did not differ on any smoking or demographic variables except race (ADHD group $=17.6 \%$ non-white; Control group $=50 \%$ non-white; $P=0.04$ ) (see Supplementary Table S1 for participant characteristics). As expected, the ADHD group had higher CAARS DSM-IV $t$-scores for Inattentive, Hyperactive/ Impulsive, and Total subscales (all $P<0.001$ ) than the control group. Within the ADHD group, $n=8$ were diagnosed with predominantly inattentive type, $n=9$ were combined type.

\section{Withdrawal and Craving}

Group $\times$ Condition interactions were observed for Craving, $\mathrm{F}(2,72)=5.73, P<0.005$ (Figure 2a) and Habit Withdrawal, $\mathrm{F}(2,72)=3.32, P<0.05$ (Supplementary Figure S1), due to the ADHD group reporting higher symptom levels in the ABS+PLAC condition compared with the Control group. See Supplementary Information for additional details on withdrawal and CO findings. 

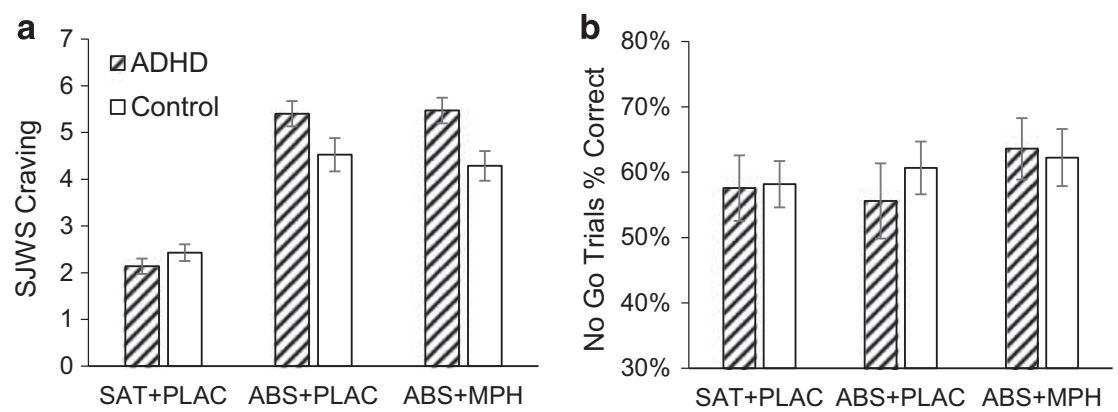

Figure 2 Prescan ratings of craving on the Shiffman-Jarvik Withdrawal Scale (SJWS) (a) and No-Go trial accuracy (b) by group (ADHD, Control) and condition (SAT+PLAC, ABS+MPH, ABS+PLAC). (a) A significant group-by-condition interaction was observed; ADHD smokers reported greater craving than Controls during ABS+PLAC, $t(72)=2.23, P<0.05$, and $A B S+M P H, t(72)=3.01, P<0.005$, conditions. (b) A main effect of condition was observed such that No-Go accuracy was higher during ABS+MPH relative to ABS+PLAC condition; pairwise comparisons indicated a significant effect of $A B S+M P H>A B S$ + PLAC on No-Go accuracy for ADHD smokers, $t(72)=2.3, P<0.05$, but not for Control smokers, $t(72)=0.5$, NS.

\section{Task Performance}

Overall effects of task. Accuracy on GO trials was uniformly high (>90\%) across all conditions and block types (see Supplementary Table S2 for additional details). Accuracy was higher during GO blocks compared with AT blocks for both Frequent-Go, $F(1,36)=24.44, P<0.0001$, and Rare-Go Trials, $F(1,36)=26.41, P<0.0001$. A similar effect of Block Type was seen for RTs for both Frequent-Go, $F$ $(1,36)=198.18, \quad P<0.0001, \quad$ and Rare-Go trials, $F$ $(1,36)=311.16, \quad P<0.0001$, with participants responding faster during GO blocks compared with AT blocks.

Effects of smoking abstinence. No effects of condition or group were observed between ABS+PLAC and SAT+PLAC for accuracy on any trial type. Notably, RTs were slower in the ABS+PLAC compared with the SAT+PLAC for both Frequent-Go, $F(1,33)=7.67, P<0.01$, and Rare-Go trials, $F$ $(1,33)=6.23, \quad P=0.02$. Moreover, a Condition-by-BlockType interaction was observed for Frequent, $F(1,33)=6.5$, $P=0.02$ and Rare-Go RTs, $F(1,33)=5.45, P=0.03$, such that abstinence slowed RTs primarily during GO blocks. No Group or Group-by-Condition interaction were observed for RT for either Frequent or Rare-Go Trials.

Effects of methylphenidate. As shown in Figure $2 \mathrm{~b}$, we observed an effect of Condition such that accuracy was better in the ABS+MPH condition compared with the ABS+PLAC condition, $\mathrm{F}(1,36)=5.34, P<0.05$. ADHD status did not moderate this effect. RTs on Frequent-Go Trials were slower in the ABS+PLAC compared with $\mathrm{ABS}+\mathrm{MPH}$ conditions, $\mathrm{F}$ $(1,33)=4.72, P=0.04$.

\section{Neuroimaging Results}

Sustained inhibitory control. Overall Task Effects. The contrast of AT $>\mathrm{GO}$ blocks was associated with bilateral activation in multiple brain regions, including superior parietal cortex, occipital cortex (OCC), angular gyrus, prefrontal cortex (PFC) including supplementary motor area, ACC, precentral gyrus, DLPFC, and rIFG, and thalamus, putamen, anterior insula, brain stem, and cerebellum (Supplementary Table S3; Supplementary Figure S2).
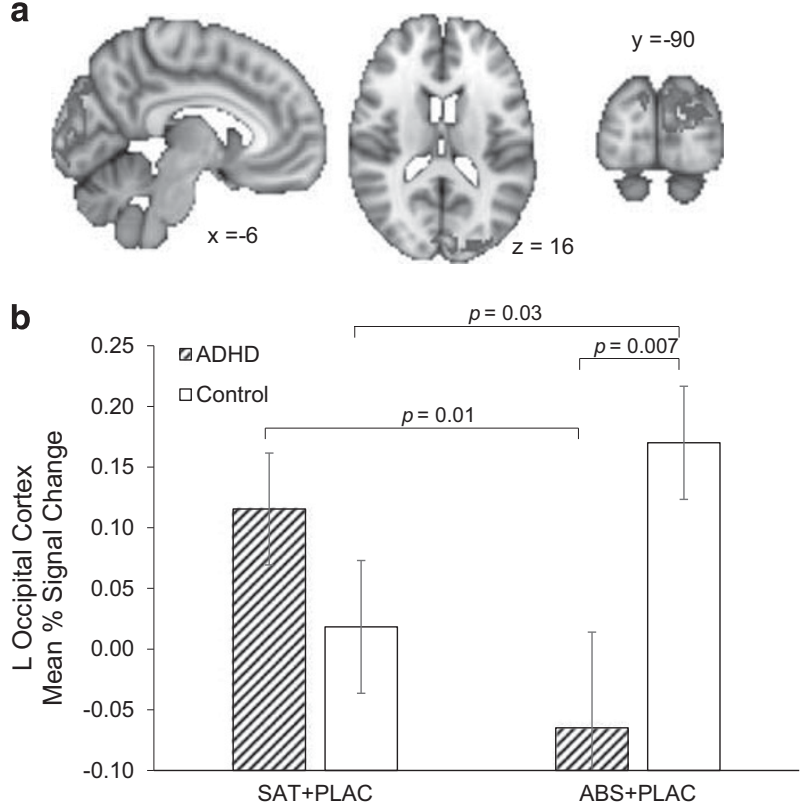

Figure 3 (a) Significant group (ADHD, Control) by abstinence condition (SAT+PLAC, ABS+PLAC) interaction for sustained inhibitory control (All Trial blocks $>$ Go Only blocks) in the left occipital cortex encompassing I I 89 voxels, $Z_{\max }=4$. I 8, peak voxel at $-22,-86,40$. (b) Mean percentage of signal change extracted from left occipital cluster, plotted by group across SAT+PLAC and ABS+PLAC conditions. Error bars represent SEM.

Effects of Smoking Abstinence. As shown in Figure 3a, a significant Group (ADHD, Control) $\times$ Condition (SAT + PLAC, ABS+PLAC) effect was observed in the left OCC $\left(Z_{\max }=4.18 ; k=1189\right.$ voxels; peak voxel $\left.=-22,-86,40\right)$, including portions of occipital pole, cuneus, and lateral superior occipital cortex extending to superior parietal lobule. Post hoc analysis of BOLD signal extracted from this region (Figure $3 \mathrm{~b}$ ) suggested that the interaction was due to lower BOLD signal in the ADHD group in the ABS+PLAC condition relative to the SAT+PLAC condition $(P=0.01)$, whereas the opposite pattern was observed for the Control group $(P<0.05)$. Moreover, within the ABS+PLAC condition, BOLD signal was lower in the ADHD compared with Control group $(P<0.01)$.

Effects of Methylphenidate. Evaluation of the effects of $\mathrm{MPH}$ on the AT>GO contrast revealed significant ABS $+\mathrm{MPH}>\mathrm{ABS}+\mathrm{PLAC}$ effects throughout bilateral superior 


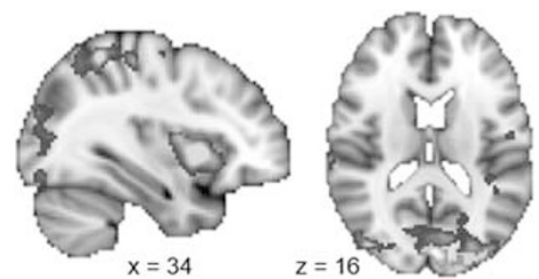

Figure 4 Whole-brain correlations between abstinence-induced changes in No-Go trial accuracy (ABS+PLAC - SAT+PLAC difference for NoGo percent correct) and abstinence-induced changes in sustained inhibitory control (ABS+PLAC - SAT+PLAC difference for All Trial > Go Only block effects), shown in blue. Significant clusters of activation were observed in the left lateral occipital cortex ( 13980 voxels, $Z_{\max }=4.65$, peak voxel at -18 , -84, 40); left planum polare/central opercular cortex (254l voxels, $Z_{\max }=3.85$, peak voxel at $\left.-56,-8,4\right)$; and right insula (I269 voxels, $Z_{\max }=4.83$, peak voxel at 32, 12,2). Activation map overlayed on occipital cortex cluster exhibiting group-by-abstinence condition interaction for sustained inhibitory control (Figure 3a), shown in red. Areas shown in green represent overlap between blue and red activation maps. A full color version of this figure is available at the Neuropsychopharmacology journal online.

parietal cortex, superior frontal gyrus, OCC, and insula $\left(Z_{\max }=4.99 ; k=50270\right.$ voxels; peak voxel $=-22,-50,68$; Supplementary Figure S3; Supplementary Table S4). No interaction between Group and MPH was observed.

Correlation with Task Performance. Given the aboveobserved effects, we evaluated correlations between abstinence or MPH-induced changes in BOLD activation and task performance. As shown in Figure 4, positive correlations between abstinence-induced changes in inhibitory control accuracy and BOLD signal were observed in the full sample in multiple brain regions, including bilateral OCC and insula, right superior parietal cortex, and temporal gyrus (Supplementary Table S5). In these brain regions, decreased accuracy during smoking abstinence was associated with decreased BOLD signal. Group differences in the pattern of correlation were not observed nor were negative correlations. No correlations were observed between MPH-induced changes in task performance and BOLD activation.

Transient inhibitory control. Task Effects. The contrast of No-Go $>$ Rare-Go trials was associated with activation in superior parietal lobule, DLPFC, precentral gyrus, IFG, and frontal pole. Activation tended to be greater in the right than the left hemisphere. Bilateral activations were also observed in OCC, precuneus, middle temporal gyrus, supplementary motor area, anterior and posterior cingulate, putamen, anterior insula, and left cerebellum (Supplementary Table S6; Supplementary Figure S4).

Effects of Smoking Abstinence. No main effects of smoking condition or interactions with group were observed.

Effects of Methylphenidate. Administration of MPH (ABS $+\mathrm{MPH}>\mathrm{ABS}+\mathrm{PLAC}$ ) resulted in increased No-Go $>$ RareGo activation in a large cluster encompassing medial PFC, orbitofrontal cortex, and subgenual ACC extending to left hippocampus/parahippocampal gyrus $\quad\left(Z_{\max }=4.08\right.$; $k=11546$ voxels; peak voxel $=14,34,-6)$ and a cluster in precuneus/posterior cingulate $\left(Z_{\max }=3.76 ; k=1286\right.$ voxels; peak voxel $=-20,-50,22$ ) (Supplementary Figure S5). Furthermore, a significant Group (ADHD, Control) by Condition (ABS+MPH, ABS+PLAC) interaction was observed in the left putamen $\left(Z_{\max }=3.19 ; k=1201\right.$ voxels; peak
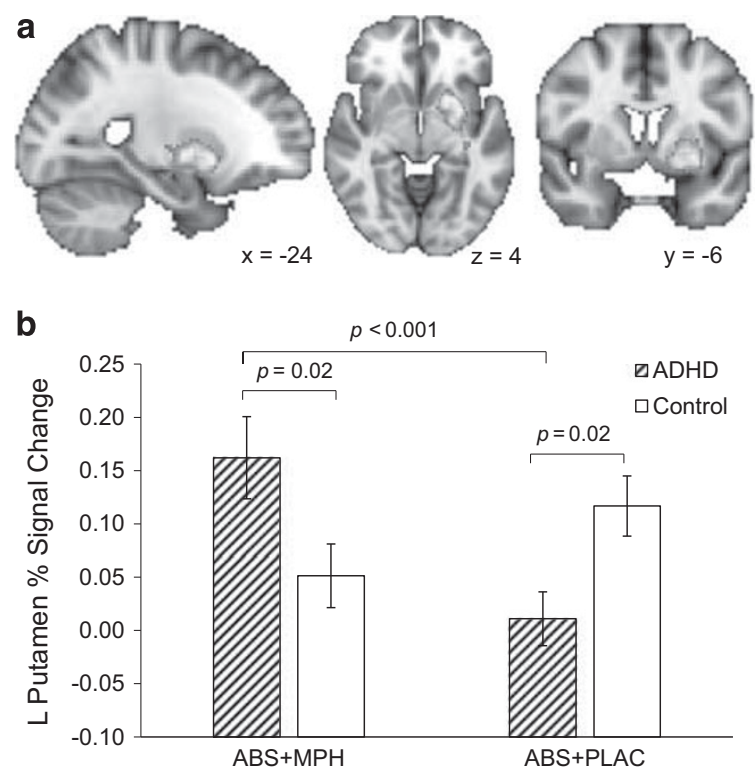

Figure 5 (a) Significant group (ADHD, Control) by methylphenidate condition (ABS+MPH, ABS+PLAC) interaction for transient inhibitory control (No-Go trials > Rare-Go trials) in left putamen encompassing I20 I voxels, $Z_{\max }=3.19$, peak voxel at $-20,10,-6$. (b) Mean percentage of signal change extracted from left putamen cluster, plotted by group across $\mathrm{ABS}+\mathrm{MPH}$ and $\mathrm{ABS}+\mathrm{PLAC}$ conditions. Error bars represent SEM.

voxel $=-20,10,-6)$ (Figure 5a). Post hoc analysis of BOLD signal extracted from this region (Figure $5 \mathrm{~b}$ ) indicated that $\mathrm{MPH}$ increased BOLD signal in the ADHD group relative to the ABS+PLAC condition $(P<0.001)$, whereas a nonsignificant trend in the opposite direction was observed for Controls $(P=0.06)$.

\section{DISCUSSION}

This study is the first to examine the effects of smoking abstinence and methylphenidate on inhibitory control among smokers with and without ADHD, allowing us to probe the neurobiological mechanisms underlying comorbidity between smoking and ADHD. Our results provided compelling preliminary evidence that abstinence from smoking is associated with decreased activation in the OCC during sustained inhibitory control among ADHD smokers compared with non-ADHD smokers. Moreover, we found that abstinence-induced decreases in OCC activation were associated with decrements in inhibitory control performance in both ADHD and non-ADHD smokers.

We hypothesized that ADHD smokers would exhibit decreased task-related activation throughout key frontoparietal regions, including rIFG and ACC, which have previously been shown to be involved in inhibitory control, during abstinence relative to both satiated and MPH conditions. Although the Go/No-Go task reliably recruited multiple regions throughout expected circuitry, group-byabstinence interaction effects were restricted to OCC-an unexpected finding. However, recruitment of OCC is frequently observed in fMRI studies of inhibitory control (Watanabe et al, 2002; Simmonds et al, 2008), and activation in this region has been proposed to be the result of top-down modulation of visual processing from fronto-parietal regions 
involved in inhibitory and attentional control (Parks and Madden, 2013). This perspective is supported by findings that attention-modulated activation of visual cortex and performance on a visual discrimination task are directly correlated with structural connectivity with fronto-parietal regions (Bennett et al, 2012; Greenberg et al, 2012). Thus, in the context of the inhibitory control task in present study, such activation may reflect increased attentional demands required for successful visual discrimination during All-Trial blocks compared with simple motor performance during GoOnly blocks.

Our results also indicated that greater abstinence-induced decrements in No-Go trial accuracy correlated with abstinence-induced attenuation of OCC activation. That is, decreased OCC activation during abstinence was directly associated with a decline in behavioral performance, across both diagnostic groups. This finding provides further support for the role of OCC in inhibitory control and attentional processes and its disruption during smoking abstinence and suggests that this should be an important area of further study.

Although not typically a primary focus of investigation in studies of $\mathrm{ADHD}$, converging evidence suggests a potential role for OCC in core attentional deficits in the disorder. Neuroimaging studies have routinely observed group differences between ADHD and control subjects in OCC activation in addition to other regions during tasks of inhibition or attention (see Castellanos and Proal, 2012 for a review). In addition, anatomical studies have observed decreased gray matter volume in primary visual cortex among adults with ADHD relative to controls (Ahrendts et al, 2011), and decreased cortical thickness across multiple regions, including $\mathrm{PFC}$ and OCC, was observed among persistent relative to remitted ADHD among adults in a 33year longitudinal study (Proal et al, 2011). Furthermore, several studies have found evidence of altered structural (Pironti et al, 2014) and functional (Cocchi et al, 2012) connectivity between OCC and frontal regions among adults with ADHD vs controls.

Long-range reciprocal connections between PFC and visual cortex have been shown to be under dopaminergic control (Noudoost and Moore, 2011), and cocaine selectively increases extracellular dopamine in visual cortical regions responsible for processing visual stimuli (Muller and Huston, 2007). In the present study, administration of MPH-which increases extracellular dopamine and norepinephrine levels via blockade of catecholamine transporters (Volkow et al, 2005; Hannestad et al, 2010)_improved behavioral performance and robustly increased inhibitory control-related brain activation for both ADHD and non-ADHD smokers, including in some OCC regions shown to be attenuated among ADHD smokers during ABS+PLAC. Together, these findings suggest the possibility that smoking withdrawal in ADHD smokers may exacerbate deficits in dopamine transmission mediating attentional control in visual cortex and that MPH may help to offset these deficits.

$\mathrm{MPH}$ administration also robustly increased transient inhibitory control-related activation throughout PFC in both groups, whereas similar effects were observed in the putamen only for ADHD smokers. These results are remarkably consistent with a previous study demonstrating robust $\mathrm{MPH}-$ induced increases in inhibitory control-related activation throughout PFC in both ADHD and control boys, but opposing effects of $\mathrm{MPH}$ in the caudate and putamen, such that striatal activation was increased by MPH in the ADHD group but decreased in the control group (Vaidya et al, 1998). Such effects may be due to MPH's affinity for norepinephrine transporter binding in the PFC, leading to increased norepinephrine transmission in these regions (Berridge et al, 2006; Engert and Pruessner, 2008). By contrast, dopaminergic effects in the striatum may vary as a function of baseline dopamine activity or underlying deficits (Vaidya et al, 1998; Engert and Pruessner, 2008). However, given nonspecific effects of MPH on catecholamine transmission, further research is needed to disentangle the contribution of specific catecholamine transmission on withdrawal or MPH effects among smokers with ADHD.

Although a main effect of smoking abstinence was not observed for No-Go accuracy, whole-brain correlation analyses revealed multiple brain areas in which decreased activation during abstinence was associated with abstinenceinduced decrements in performance. This pattern was consistent across both groups and included areas of OCC, discussed above, as well as regions including bilateral insula, right superior parietal cortex, and superior temporal gyrus. The insula, in particular, has been repeatedly implicated in studies of nicotine dependence (Janes et al, 2010; Addicott et al, 2015), as well as studies of inhibitory control (Xue et al, 2008), and has been proposed as a primary region linking interoceptive awareness and cognitive control processes. Previous studies have demonstrated an association between weaker functional connectivity within a network containing the insula and other regions involved in motor planning and cognitive control and greater likelihood of relapse during a smoking quit attempt (Janes et al, 2010; Addicott et al, 2015). Although speculative, the current results are consistent with a role for the insula in response inhibition among smokers, which may be contribute to deficits in cognitive control during withdrawal.

The present findings also raise questions about the clinical utility of $\mathrm{MPH}$ as a smoking cessation aid. Our results suggested that nicotine withdrawal and MPH have opposing neuromodulatory effects, but we observed a lack of robust effects of MPH on subjective craving and withdrawal. These findings are consistent with the results of recent randomized controlled trials evaluating $\mathrm{MPH}$ and another stimulant (lisdexamfetamine dimesylate), which have observed negative results on smoking outcomes despite improvements in ADHD symptoms (Winhusen et al, 2010; Kollins et al, 2014). However, individual differences in efficacy of $\mathrm{MPH}$ as a smoking cessation adjunct have been observed among ADHD smokers (Covey et al, 2011), raising the possibility that $\mathrm{MPH}$ might improve inhibitory control and smoking outcomes among a subset of ADHD smokers, potentially those with greater disruptions in visual attention. Furthermore, it is worth noting that all of these studies included concurrent treatment with nicotine patch, whereas our study did not examine effects of MPH under satiated conditions. Thus it is unclear whether $\mathrm{MPH}$ may improve outcomes in a subset of ADHD smokers attempting to quit without the aid of nicotine replacement.

The present study had several strengths, including evaluation of neurocognitive function across three separate pharmacological conditions, comprehensive diagnostic 
assessment for both ADHD and control groups, and use of medication wash-out period prior to testing. However, several limitations are also noted. Given the clinical sample and use of repeated sessions, sample size within each group was relatively small, limiting conclusions that can be drawn without further replication. Sample size also prevented examining the effects of medication treatment history, which could have contributed to residual effects in the ADHD group despite the medication wash-out period. Unintended group differences in racial composition presented an additional potential confound. We did not examine ADHD subtypes or sex differences, which may be important considerations for future studies, particularly given the moderating role of ovarian hormones in nicotine reinforcement and ADHD symptoms (Van Voorhees et al, 2012). Finally, our study design did not allow us to examine doseresponse effects of MPH or effects of prolonged exposure, and lack of a satiation $+\mathrm{MPH}$ condition prevented determination of whether MPH effects were specific to abstinence or reflected drug effects more broadly.

Although beyond the scope of the present analyses, an important future direction will be to determine whether differences in visual attention in OCC are reflective of broader differences in functional connectivity with frontoparietal regions involved in cognitive control. Furthermore, it will be important to evaluate whether the effects of abstinence on neural markers of inhibitory control generalize to smoking-related contexts or are predictive of cessation outcomes in this patient population. For example, diminished activation in OCC during attentional control of visual stimulus processing may lead to greater attentional bias to smoking cues (Janes et al, 2010), thereby predisposing ADHD smokers to relapse. It is important to note that the extent to which response inhibition in the context of the $\mathrm{Go} /$ No-Go task relates to inhibitory control and decision-making in real-world contexts is unclear. However, recent work has demonstrated an association between BOLD activation during the Go/No-Go task and ability to refrain from smoking in both laboratory and real-world settings (Froeliger et al, 2017), suggesting an important link with clinical outcomes. Additional research is needed to clarify how common or dissociable mechanisms underlying different aspects of inhibitory control confer vulnerability to smoking among individuals with ADHD.

Overall, our findings provide important preliminary evidence on potential neurobiological mechanisms underlying comorbidity between smoking and ADHD. Specifically, abstinence from smoking is associated with decreased activation in OCC during sustained inhibition among ADHD smokers compared with non-ADHD smokers that is associated with inhibitory control performance. These findings advance our understanding of ADHD-smoking comorbidity and suggest important areas for future investigation, including replication in a larger sample, functional connectivity analysis, and examining association between neurocognitive mechanisms and smoking behavior.

\section{FUNDING AND DISCLOSURE}

This research was supported by NIH grants R01 DA024838 (to FJM), K24 DA023464 (to SHK), K23 DA042898 (to JAO), and K23 DA039294 (to MMS). SHK has received research support and/or consulting fees from the following commercial sources in the past 24 months: Purdue Canada, Akili Interactive, Alcobra, Bose, Jazz, Ironshore, Medgenics, Sunovion, Rhodes, Shire, SK Life Sciences, KemPharm, and NLS Pharma. The other authors declare no conflict of interest.

\section{REFERENCES}

Addicott MA, Sweitzer MM, Froeliger B, Rose JE, McClernon FJ (2015). Increased functional connectivity in an insula-based network is associated with improved smoking cessation outcomes. Neuropsychopharmacology 40: 2648-2656.

Ahrendts J, Rusch N, Wilke M, Philipsen A, Eickhoff SB, Glauche V et al (2011). Visual cortex abnormalities in adults with ADHD: a structural MRI study. World J Biol Psychiatry 12: 260-270.

Ashare RL, Falcone M, Lerman C (2014). Cognitive function during nicotine withdrawal: Implications for nicotine dependence treatment. Neuropharmacology 76(Pt B): 581-591.

Ashare RL, Hawk LW Jr. (2012). Effects of smoking abstinence on impulsive behavior among smokers high and low in ADHD-like symptoms. Psychopharmacology 219: 537-547.

Barkley RA (1997). Behavioral inhibition, sustained attention, and executive functions: constructing a unifying theory of ADHD. Psychol Bull 121: 65-94.

Bennett IJ, Motes MA, Rao NK, Rypma B (2012). White matter tract integrity predicts visual search performance in young and older adults. Neurobiol Aging 33: 433 e421-433.e431.

Berridge CW, Devilbiss DM, Andrzejewski ME, Arnsten AF, Kelley AE, Schmeichel B et al (2006). Methylphenidate preferentially increases catecholamine neurotransmission within the prefrontal cortex at low doses that enhance cognitive function. Biol Psychiatry 60: 1111-1120.

Castellanos FX, Proal E (2012). Large-scale brain systems in ADHD: beyond the prefrontal-striatal model. Trends Cogn Sci 16: 17-26.

Chikazoe J, Jimura K, Asari T, Yamashita K, Morimoto H, Hirose S et al (2009). Functional dissociation in right inferior frontal cortex during performance of go/no-go task. Cereb Cortex 19: 146-152.

Cocchi L, Bramati IE, Zalesky A, Furukawa E, Fontenelle LF, Moll J et al (2012). Altered functional brain connectivity in a nonclinical sample of young adults with attention-deficit/hyperactivity disorder. J Neurosci 32: 17753-17761.

Congdon E, Mumford JA, Cohen JR, Galvan A, Aron AR, Xue G et al (2010). Engagement of large-scale networks is related to individual differences in inhibitory control. Neuroimage 53: 653-663.

Covey LS, Hu MC, Weissman J, Croghan I, Adler L, Winhusen T (2011). Divergence by ADHD subtype in smoking cessation response to OROS-methylphenidate. Nicotine Tob Res 13: 1003-1008.

Cubillo A, Halari R, Smith A, Taylor E, Rubia K (2012). A review of fronto-striatal and fronto-cortical brain abnormalities in children and adults with Attention Deficit Hyperactivity Disorder (ADHD) and new evidence for dysfunction in adults with ADHD during motivation and attention. Cortex 48: 194-215.

Dillo W, Goke A, Prox-Vagedes V, Szycik GR, Roy M, Donnerstag F et al (2010). Neuronal correlates of ADHD in adults with evidence for compensation strategies-a functional MRI study with a Go/ No-Go paradigm. Ger Med Sci 8: Doc09.

Engert V, Pruessner JC (2008). Dopaminergic and noradrenergic contributions to functionality in ADHD: the role of methylphenidate. Curr Neuropharmacol 6: 322-328.

Froeliger B, McConnell PA, Bell S, Sweitzer M, Kozink RV, Eichberg C et al (2017). Association between baseline corticothalamic-mediated inhibitory control and smoking relapse vulnerability. JAMA Psychiatry 74: 379-386. 
Ghahremani DG, Lee B, Robertson CL, Tabibnia G, Morgan AT, De Shetler $\mathrm{N}$ et al (2012). Striatal dopamine $\mathrm{D}(2) / \mathrm{D}(3)$ receptors mediate response inhibition and related activity in frontostriatal neural circuitry in humans. J Neurosci 32: 7316-7324.

Greenberg AS, Verstynen T, Chiu YC, Yantis S, Schneider W, Behrmann M (2012). Visuotopic cortical connectivity underlying attention revealed with white-matter tractography. J Neurosci 32: 2773-2782.

Hannestad J, Gallezot JD, Planeta-Wilson B, Lin SF, Williams WA, van Dyck $\mathrm{CH}$ et al (2010). Clinically relevant doses of methylphenidate significantly occupy norepinephrine transporters in humans in vivo. Biol Psychiatry 68: 854-860.

Hart H, Radua J, Nakao T, Mataix-Cols D, Rubia K (2013). Metaanalysis of functional magnetic resonance imaging studies of inhibition and attention in attention-deficit/hyperactivity disorder: exploring task-specific, stimulant medication, and age effects. JAMA Psychiatry 70: 185-198.

Humfleet GL, Prochaska JJ, Mengis M, Cullen J, Munoz R, Reus V et al (2005). Preliminary evidence of the association between the history of childhood attention-deficit/hyperactivity disorder and smoking treatment failure. Nicotine Tob Res 7: 453-460.

Janes AC, Pizzagalli DA, Richardt S, Frederick Bde B, Holmes AJ, Sousa J et al (2010). Neural substrates of attentional bias for smoking-related cues: an FMRI study. Neuropsychopharmacology 35: 2339-2345.

Kessler RC, Adler L, Barkley R, Biederman J, Conners CK, Demler $\mathrm{O}$ et al (2006). The prevalence and correlates of adult ADHD in the United States: results from the National Comorbidity Survey Replication. Am J Psychiatry 163: 716-723.

Kollins SH, English JS, Itchon-Ramos N, Chrisman AK, Dew R, O'Brien B et al (2014). A pilot study of lis-dexamfetamine dimesylate (LDX/SPD489) to facilitate smoking cessation in nicotine-dependent adults with ADHD. J Atten Disord 18: $158-168$

Kollins SH, English JS, Roley ME, O'Brien B, Blair J, Lane SD et al (2013). Effects of smoking abstinence on smoking-reinforced responding, withdrawal, and cognition in adults with and without attention deficit hyperactivity disorder. Psychopharmacology 227: 19-30.

Kozink RV, Kollins SH, McClernon FJ (2010). Smoking withdrawal modulates right inferior frontal cortex but not presupplementary motor area activation during inhibitory control. Neuropsychopharmacology 35: 2600-2606.

McClernon FJ, Addicott MA, Sweitzer MM (2015). Smoking abstinence and neurocognition: implications for cessation and relapse. Curr Top Behav Neurosci 23: 193-227.

McClernon FJ, Kollins SH (2008a). ADHD and smoking: from genes to brain to behavior. Ann NY Acad Sci 1141: 131-147.

McClernon FJ, Kollins SH, Lutz AM, Fitzgerald DP, Murray DW, Redman C et al (2008b). Effects of smoking abstinence on adult smokers with and without attention deficit hyperactivity disorder: results of a preliminary study. Psychopharmacology 197: 95-105.

McClernon FJ, Van Voorhees EE, English J, Hallyburton M, Holdaway A, Kollins SH (2011). Smoking withdrawal symptoms are more severe among smokers with ADHD and independent of ADHD symptom change: results from a 12-day contingencymanaged abstinence trial. Nicotine Tob Res 13: 784-792.

Milberger S, Biederman J, Faraone SV, Chen L, Jones J (1997). ADHD is associated with early initiation of cigarette smoking in children and adolescents. J Am Acad Child Adolesc Psychiatry 36: 37-44.

Molina BS, Pelham WE Jr. (2003). Childhood predictors of adolescent substance use in a longitudinal study of children with ADHD. J Abnorm Psychol 112: 497-507.
Muller CP, Huston JP (2007). Dopamine activity in the occipital and temporal cortices of rats: dissociating effects of sensory but not pharmacological stimulation. Synapse 61: 254-258.

Noudoost B, Moore T (2011). Control of visual cortical signals by prefrontal dopamine. Nature 474: 372-375.

Parks EL, Madden DJ (2013). Brain connectivity and visual attention. Brain Connect 3: 317-338.

Pironti VA, Lai MC, Muller U, Dodds CM, Suckling J, Bullmore ET et al (2014). Neuroanatomical abnormalities and cognitive impairments are shared by adults with attention-deficit/hyperactivity disorder and their unaffected first-degree relatives. Biol Psychiatry 76: 639-647.

Pomerleau OF, Downey KK, Stelson FW, Pomerleau CS (1995). Cigarette smoking in adult patients diagnosed with attention deficit hyperactivity disorder. J Subst Abuse 7: 373-378.

Powell J, Dawkins L, West R, Powell J, Pickering A (2010). Relapse to smoking during unaided cessation: clinical, cognitive and motivational predictors. Psychopharmacology 212: 537-549.

Proal E, Reiss PT, Klein RG, Mannuzza S, Gotimer K, RamosOlazagasti MA et al (2011). Brain gray matter deficits at 33-year follow-up in adults with attention-deficit/hyperactivity disorder established in childhood. Arch Gen Psychiatry 68: 1122-1134.

Shiffman SM, Jarvik ME (1976). Smoking withdrawal symptoms in two weeks of abstinence. Psychopharmacology 50: 35-39.

Simmonds DJ, Pekar JJ, Mostofsky SH (2008). Meta-analysis of Go/Nogo tasks demonstrating that fMRI activation associated with response inhibition is task-dependent. Neuropsychologia 46: 224-232.

Vaidya CJ, Austin G, Kirkorian G, Ridlehuber HW, Desmond JE, Glover GH et al (1998). Selective effects of methylphenidate in attention deficit hyperactivity disorder: a functional magnetic resonance study. Proc Natl Acad Sci USA 95: 14494-14499.

Van Voorhees EE, Mitchell JT, McClernon FJ, Beckham JC, Kollins SH (2012). Sex, ADHD symptoms, and smoking outcomes: an integrative model. Med Hypotheses 78: 585-593.

Volkow ND, Wang GJ, Fowler JS, Ding YS (2005). Imaging the effects of methylphenidate on brain dopamine: new model on its therapeutic actions for attention-deficit/hyperactivity disorder. Biol Psychiatry 57: 1410-1415.

Volkow ND, Wang GJ, Kollins SH, Wigal TL, Newcorn JH, Telang F et al (2009). Evaluating dopamine reward pathway in ADHD: clinical implications. JAMA 302: 1084-1091.

Volkow ND, Wang GJ, Newcorn J, Telang F, Solanto MV, Fowler JS et al (2007). Depressed dopamine activity in caudate and preliminary evidence of limbic involvement in adults with attention-deficit/hyperactivity disorder. Arch Gen Psychiatry 64: 932-940.

Watanabe J, Sugiura M, Sato K, Sato Y, Maeda Y, Matsue Y et al (2002). The human prefrontal and parietal association cortices are involved in NO-GO performances: an event-related fMRI study. Neuroimage 17: 1207-1216.

Willcutt EG (2012). The prevalence of DSM-IV attention-deficit/ hyperactivity disorder: a meta-analytic review. Neurotherapeutics 9: $490-499$.

Winhusen TM, Somoza EC, Brigham GS, Liu DS, Green CA, Covey LS et al (2010). Impact of attention-deficit/hyperactivity disorder (ADHD) treatment on smoking cessation intervention in ADHD smokers: a randomized, double-blind, placebo-controlled trial. $J$ Clin Psychiatry 71: 1680-1688.

Xue G, Aron AR, Poldrack RA (2008). Common neural substrates for inhibition of spoken and manual responses. Cereb Cortex 18: 1923-1932.

Zhang L, Dong Y, Doyon WM, Dani JA (2012). Withdrawal from chronic nicotine exposure alters dopamine signaling dynamics in the nucleus accumbens. Biol Psychiatry 71: 184-191.

Supplementary Information accompanies the paper on the Neuropsychopharmacology website (http://www.nature.com/npp) 